\title{
Theoretical modeling and analysis of the emission spectra of a ChemCam standard: Basalt BIR1
}

\author{
J. Colgan ${ }^{a}$, E. J. Judge ${ }^{b}$, H. M. Johns ${ }^{a}$, D. P. Kilcrease ${ }^{a}$, J. E. Barefield $\mathrm{II}^{b}$, \\ R. McInroy ${ }^{c}$, P. Hakel ${ }^{d}$, R. C. Wiens ${ }^{e}$, and S. M. Clegg ${ }^{c}$ \\ ${ }^{a}$ Theoretical Division, Los Alamos National Laboratory, Los Alamos, NM 87545 \\ ${ }^{b}$ Chemical Diagnostics and Engineering, Los Alamos National Laboratory, Los Alamos, \\ NM 87545 \\ ${ }^{c}$ Physical Chemistry and Applied Spectroscopy, Los Alamos National Laboratory, Los \\ Alamos, NM 87545 \\ ${ }^{d}$ Computational Physics Division, Los Alamos National Laboratory, Los Alamos, NM \\ 87545 \\ e Space and Remote Sensing Division, Los Alamos National Laboratory, Los Alamos, NM \\ 87545
}

\begin{abstract}
We report on efforts to perform theoretical modeling of the emission spectrum measured from a basalt sample. We compare our calculations with measurements that were made to provide standards for the ChemCam instrument on the Mars Science Laboratory. We find that to obtain good agreement between modeling and the measurement, it is necessary to determine atomic and ionic level populations via a multi-element approach in which the free electron density that is created influences all the species within the plasma. Calculations that consider each element separately are found to be in poorer agreement with the measured spectrum, indicating that the 'matrix effect' term often used to describe the influence of other species on the emission spectrum from a given element is due to the influence of the global electron density of the plasma. We explore the emission features in both the visible and near-infrared wavelength ranges, and also examine radiation transport effects for some of the most intense features found in the basalt spectrum. Finally, we also provide comparisons of the ChemCam measurement with new high-resolution spectral measurements.
\end{abstract}

Keywords: LIBS, matrix effects, radiation transport, atomic physics, LTE modeling 


\section{Introduction}

The study of laser-induced plasmas, and in particular laser-induced breakdown spectroscopy (LIBS), continues to be an important tool in remote analysis and in the determination of the elemental composition of a given sample. The uses of LIBS are extremely varied and widespread and several recent reviews succinctly describe progress in the field ${ }^{1,2,3,4,5,6}$. An issue that is periodically discussed in the analysis of LIBS plasmas is the so-called 'matrix effects' phenomenon. This term has been used to describe the observation that the emission spectrum generated by LIBS can be modified by the other elements present in the sample. For example, Vrenegor et $\mathrm{al}^{7}$ examine matrix effects in LIBS plasmas generated from various high-alloy steel samples. In earlier work, Iida ${ }^{8}$ examined the effects of the atmosphere on the amount of vaporized sample in different atmospheric pressures and for different atmospheric gases, and Henry et $\mathrm{al}^{9}$ investigated the role of helium addition in LIBS analysis of $\mathrm{C}$ and $\mathrm{H}$ emission. A significant decrease in electron density with increasing helium addition was reported. Diwakar et $\mathrm{al}^{10}$ analyzed matrix effects in LIBS plasmas formed from multi-component aerosol particles. Significant enhancement was observed of the emission lines of $\mathrm{Na}$ when $\mathrm{Cu}$ was added to the aerosol analyte, and the intensity ratio of several emission lines from $\mathrm{Cd}$ was found to increase with increasing $\mathrm{Cd}$ mass fraction within an aersol composed of $\mathrm{Mg}$ and $\mathrm{Cd}$. Tognoni et al ${ }^{11}$ proposed a diagnostic tool (valid for local thermodynamic equilibrium) for multi-element plasmas that depends on a 'double ratio' of the line intensities from two test elements. This tool was used to analyze the temperature of a plasma formed from $\mathrm{Mg}$ and $\mathrm{Zn}$ solutions in the presence of a Ar nebulizer carrier gas. More recently, Aguilera et al ${ }^{12}$ explored matrix effects in LIBS plasmas generated from various metallic samples that included $\mathrm{Fe}, \mathrm{Ni}, \mathrm{Cu}$, and $\mathrm{Al}$. A recent study by Dong et al $^{13}$ also explored the emission spectra from LIBS plasmas of coal in different gas environments, with the most intense lines observed when the sample was in an Ar atmosphere. One of the main goals of the work reported here is to provide a potential physics explanation of some of these matrix effects that have been reported.

LIBS is the remote sensing tool that is used by the ChemCam instrument on the Mars Science Laboratory rover Curiosity ${ }^{14}$. ChemCam has been very successful in providing many datasets of Martian geological samples. Within its $7 \mathrm{~m}$ reach and among the 200,000 spectra provided as of early 2015, the ChemCam LIBS tool has explored stratigraphic layers of sedimentary rock 
outcrops, secondary alteration features found in veins and mm-size ridges, and has sampled coarse-grained minerals in igneous rocks ${ }^{15}$. Before deployment of ChemCam on Mars, extensive calibration measurements were performed $^{16,17}$, and a library of geologic standards ${ }^{18,19,20}$ was developed to assist in the analysis of the spectra obtained from ChemCam on Mars. The initial LIBS spectral library ${ }^{20,21}$ consisted of 69 geologic samples from which LIBS plasmas were generated under Martian conditions using a ChemCam instrument. Experiments were performed at different distances from the ChemCam mast unit to the target and for various other different experimental arrangements. Spectra were collected in three different wavelength bands that spanned 240-850 nm. Due to the easy access to these standards, we decided to attempt theoretical modeling of the BIR1 basalt sample (see Table 1) from the ChemCam library ${ }^{20}$. This study would serve two purposes; it would allow a determination of how well one could theoretically predict emission spectra measured using ChemCam, and it would enable exploration of 'matrix effects' in such measurements. We also consider the effects of radiation transport within the LIBS plasmas analyzed here. Plasma inhomogeneities and radiation transport effects have previously been examined by Wester and Noll $^{22}$. The evolution of laser-induced plasma has been studied by Casavola et $\mathrm{al}^{23}$ and dynamics in laser-induced plasmas has also been investigated by Surzhikov et $\mathrm{al}^{24}$. Kinetic processes in laser-induced plasmas were also discussed by Pietanza et $a^{25}$. Early-time (in the first 100s of ns) plasma dynamics have also been investigated by Diwakar and Hahn ${ }^{26}$, and the evolution of the plasma dynamics for a laser-induced plasma expanding into a vacuum was explored by Gornushkin et $\mathrm{al}^{27}$. The plasma dynamics after laser ablation has recently been investigated by Harilal et $\mathrm{al}^{28}$ and Hussein et $\mathrm{al}^{29}$. These hydrodynamic studies explored the plume expansion of the plasma and the models were in reasonable agreement with measurements. Very few studies have attempted to theoretically simulate the measured spectra from LIBS targets. Our modeling considers all of the major elements present in the ChemCam BIR1 sample, and we also considered the effects of the $\mathrm{CO}_{2}$ atmosphere in which the measurements were performed. We perform such theoretical modeling using the Los Alamos ATOMIC code ${ }^{30,31}$ that was recently used to analyze the emission spectrum from a LIBS plasma produced from iron oxide.

The paper is organized as follows. Section 2 provides a brief discussion of the measurements used to generate the emission spectra presented in this paper, including the composition of the basalt sample under investiga- 
tion. Section 3 provides a detailed description of our theoretical approach to the modeling of a plasma generated from basalt. In Section 4 we present a detailed comparison of the ChemCam basalt standard with our theoretical calculations and discuss the influence of matrix effects. In Section 5 we present new measurements of the emission spectrum from basalt using a high-resolution spectrometer, and compare and contrast these to the measurements in the ChemCam library. We end with a short summary and outlook for future work.

\section{Experimental Set-up and ChemCam Standards}

The ChemCam instrument used in the following experiments has been previously described in detail ${ }^{20,21}$. Briefly, the laser system, designed and built by CNES and Thales Optronics, consists of a passively-cooled diodepumped Nd:KGW laser with two amplifier slabs producing 5 ns pulses at $1067 \mathrm{~nm}$ at a repetition rate of $3 \mathrm{~Hz}$ with energies up to $14 \mathrm{~mJ}$ on target when operated between -10 and $+10{ }^{\circ} \mathrm{C}$. The generated beam is expanded and then focused through a $110 \mathrm{~mm}$ diameter SchmidtCassegrain telescope to produce an approximately $400 \mu \mathrm{m}$ ablation spot on samples at a distance of 1.6 meters. The same telescope collects the plasma light, focusing it into an optical fiber leading to a demultiplexer and three spectrometers covering 240-850 nm, except for a gap from 340-385 nm. The spectrometers, referred to as UV, violet (VIO), and visible and near-infrared (VNIR) are crossed Czerny-Turner designs each equipped with a e2v 42-10 back illuminated CCD. Integration times are approximately $10 \mathrm{~ms}$. Samples were analyzed within a vacuum chamber containing $930 \pm 130 \mathrm{~Pa}$ of $\mathrm{CO}_{2}$ to simulate the Martian atmosphere.

Higher spectral resolution ( $\sim 10,000$ resolving power) measurements (shown in Section 5) were acquired using a Catalina Scientific EMU-65 UV/VIS/NIR Echelle Spectrometer equipped with a UV3 cassette. The spectrometer was coupled to the ChemCam mast unit via the fiber output of the telescope described above. The same laser parameters, vacuum chamber conditions, and sample were used. To mimic the ChemCam acquisition parameters, no timing controls (delay time or gate width) were implemented. An integration time of three seconds and six accumulations yielded approximately 54 laser shot acquisition.

The sample used was a basalt, BIR-1A, from the United States Geological Survey. The composition of the major components of this sample is given in 
Table 1: Composition (in weight percentage of the oxide) of the BIR1 basalt sample from which a LIBS plasma was created and analyzed using ChemCam as described in ${ }^{20}$.

\begin{tabular}{lllllllllll}
\hline Name & $\mathrm{SiO}_{2}$ & $\mathrm{TiO}_{2}$ & $\mathrm{Al}_{2} \mathrm{O}_{3}$ & $\mathrm{Fe}_{2} \mathrm{O}_{3}$ & $\mathrm{MnO}$ & $\mathrm{MgO}$ & $\mathrm{CaO}$ & $\mathrm{Na}_{2} \mathrm{O}$ & $\mathrm{K}_{2} \mathrm{O}$ & $\mathrm{P}_{2} \mathrm{O}_{3}$ \\
\hline BIR1 & 47.70 & 0.97 & 15.40 & 11.33 & 0.18 & 9.70 & 13.4 & 1.81 & 0.03 & 0.03 \\
\hline
\end{tabular}

Table 1 (where we note that contributions from trace elements are neglected in the analysis presented here). Approximately 5 grams of powdered BIR-1A was pressed for 5 minutes at 10 Tons producing a $25 \mathrm{~mm}$ x $5 \mathrm{~mm}$ disc. The sample was placed in the vacuum chamber and analyzed without further preparation or handling.

\section{Theoretical Modeling}

Our atomic data calculations were performed for all the elements listed in Table 1 in a similar manner to the calculations performed in our recent study of Fe plasma ${ }^{31}$. That is, the Los Alamos semi-relativistic atomic structure code CATS $^{32,33}$ was used to generate wavefunctions and fine-structure energy levels for the neutral and singly-ionized species of interest. Transition probabilities and plane-wave-Born (PWB) collision strengths were also generated. As previously described in some detail, we also performed a shift of our computed energy levels to their known NIST values ${ }^{34}$. The multi-purpose ionization code GIPPER ${ }^{35}$ is then used to compute all relevant ionization cross sections. The number of configurations and resulting fine-structure energy levels generated for each neutral atom and singly ionized ion are listed in Table 2. The differing number of levels used for each atomic species reflects the complexity of the atomic structure of these elements. Systems in which the lowest energetic configurations contain multiple electrons in partiallyfilled subshells (for example, Mn) spawn large numbers of levels compared to systems in which the lowest energy configurations are much simpler (such as $\mathrm{Al}$ ). Several of these elements were found to retain almost all of their population in the singly ionized ion under the conditions found in the LIBS plasma; we checked that adding the twice-ionized ion to the atomic model for such elements made almost no difference to the modeling results presented in this paper.

We use the plasma kinetics code ATOMIC to perform the calculations that produce level populations and resulting emission spectra. ATOMIC may be run in LTE or non-LTE mode (with the latter significantly more 
Table 2: Number of configurations (ncfg) and number of levels (nlev) included in the ATOMIC modeling of the LIBS plasma created from basalt.

\begin{tabular}{llllllllll}
\hline Name & $\mathrm{Si}$ & $\mathrm{Si}^{+}$ & $\mathrm{Ti}$ & $\mathrm{Ti}^{+}$ & $\mathrm{Al}$ & $\mathrm{Al}^{+}$ & $\mathrm{Fe}$ & $\mathrm{Fe}^{+}$ & \\
\hline ncfg & 52 & 51 & 42 & 47 & 46 & 48 & 28 & 27 \\
nlev & 1046 & 311 & 6543 & 2087 & 255 & 213 & 12797 & 15538 & \\
\hline \hline Name & $\mathrm{Mn}$ & $\mathrm{Mn}^{+}$ & $\mathrm{Mg}$ & $\mathrm{Mg}^{+}$ & $\mathrm{Ca}$ & $\mathrm{Ca}^{+}$ & $\mathrm{Na}$ & $\mathrm{Na}^{+}$ & \\
\hline ncfg & 38 & 38 & 78 & 42 & 85 & 37 & 46 & 43 & \\
nlev & 17628 & 16268 & 526 & 239 & 783 & 103 & 311 & 786 & \\
\hline \hline Name & $\mathrm{K}$ & $\mathrm{K}^{+}$ & $\mathrm{P}$ & $\mathrm{P}^{+}$ & $\mathrm{O}$ & $\mathrm{O}^{+}$ & $\mathrm{C}$ & $\mathrm{C}^{+}$ & $\mathrm{H}$ \\
\hline ncfg & 49 & 50 & 56 & 55 & 69 & 69 & 69 & 75 & 55 \\
nlev & 855 & 2294 & 2375 & 1535 & 3543 & 3174 & 1891 & 882 & 100
\end{tabular}

computationally intensive). To model the spectrum produced from basalt, we include all the elements for the species described in Table 1, with the same relative abundances. Since the overall mass of the ablated plasma is not known, we multiply all the mass densities that result from the abundances listed in Table 1 by $10^{-6} \mathrm{~g} / \mathrm{cm}^{3}$ which results in a total mass that is reasonably consistent with estimates of the mass ablated in a LIBS measurement ${ }^{36}$. The actual mass densities used in the calculations presented in the following section are listed in Table 3 .

At this point in our modeling some assumptions are necessary about the plasma formed from the basalt sample. For example, the basalt emission spectrum that was presented in ${ }^{20}$ showed a weak $\mathrm{H}$ line at $656 \mathrm{~nm}$, indicating that the sample likely contained an unknown amount of water at the time of the LIBS measurement. We therefore need to include water in our model (which is achieved by adding $\mathrm{H}$ and $\mathrm{O}$ at the appropriate relative abundances). Also, it is probable that some of the $\mathrm{CO}_{2}$ atmosphere will couple into the plasma, but it is unknown how much. Subsequently we added C and $\mathrm{O}$, again with the appropriate relative abundances, to the calculations presented here.

The ATOMIC calculations presented here use an iterative mixing procedure $^{37}$ to obtain the emissivity of the plasma. The iterative mixing approach was first used for stellar opacities ${ }^{38,39}$. This set of calculations begins with the mass density for each species, at a given electron temperature. Initially, a single-element atomic model is solved that returns an electron density for each element as well as the average ionization and set of level populations of 
Table 3: Mass density (in $10^{-6} \mathrm{~g} / \mathrm{cm}^{3}$ ) of the elements included in our LIBS plasma modeling. Hydrogen is included because of the likelihood that the BIR1 sample was partially hydrated, and carbon was included due to the possibility of the presence of atmospheric constituents in the LIBS plasma.

\begin{tabular}{llllllll}
\hline Name & $\mathrm{Si}$ & $\mathrm{Ti}$ & $\mathrm{Al}$ & $\mathrm{Fe}$ & $\mathrm{Mn}$ & $\mathrm{Mg}$ & $\mathrm{Ca}$ \\
\hline Mass density & 0.223 & 0.00581 & 0.0815 & 0.07924 & 0.00139 & 0.0585 & 0.0958 \\
\hline \hline Name & $\mathrm{Na}$ & $\mathrm{K}$ & $\mathrm{P}$ & $\mathrm{O}$ & $\mathrm{C}$ & $\mathrm{H}$ & \\
\hline Mass density & 0.01343 & 0.000249 & 0.000131 & 2.232 & 0.671 & 0.00671 &
\end{tabular}

each species at this density. The electron density and the average ionization of each species is then used to compute a new, global, electron density, which is then sent back to the single element atomic model for each species. This is then used to compute a new average ionization and set of populations for each species. This process is continued until self-consistency is achieved, i.e., until the fractional populations of each level of each element vary within a prescribed tolerance from the previous iteration.

The ATOMIC calculations presented here were performed in LTE within this multi-element framework. This mixing model should be appropriate ${ }^{40}$ at the temperatures and moderate densities found in LIBS plasmas where electron-atom and electron-ion collisions dominate. As discussed in the next section, non-LTE multi-element calculations may also be performed, although these quickly become computationally intensive when considering many species within the plasma. The ATOMIC calculations presented here include collisional broadening based on the work of Dimitrijević and Konjević $^{41}$ and also include the instrumental resolution of the spectrometer.

\subsection{Radiation transport}

We model the LIBS plasma as a series of concentric spherical shells, each at a different uniform density and temperature and centered at the origin ${ }^{42}$. We envision the plasma as somewhat hotter and denser in the inner regions and cooler and less dense in the outer regions. However, quantitative descriptions of the exact temperature and density profiles require hydrodynamics modeling, which is beyond the scope of the current work We can follow radiation passing through each shell along the chord length between the rear surface of the sphere and the detector. Each such radiation path is termed a line of sight through the plasma. If the detector is located at some distance $z_{d}$ on the $z$ axis then the chord length of a line of sight of radiation passing 
through the sphere of radius $r_{2}$ is given by $L_{a d}(y)=2 \sqrt{r_{2}^{2}-y^{2} /\left(1+y^{2} / z_{d}^{2}\right)}$ where $y$ is perpendicular to the $z$ axis at the origin (see figure 1 ). In this case we have $z_{d}>>r_{2}$ so that $L_{a d}(y) \approx 2 \sqrt{r_{2}^{2}-y^{2}}$ and all lines of sight can be regarded as parallel to the $z$ axis with the specific intensity of radiation $I_{\nu}(s)$ for frequency $\nu$ at distance $s$ along a single chord given by the following solution to the radiative transfer equation ${ }^{42}$

$$
I_{\nu}(s)=I_{\nu}(s=0) e^{-\alpha_{\nu} s}+\frac{\epsilon_{\nu}}{\alpha_{\nu}}\left(1-e^{-\alpha_{\nu} s}\right)
$$

where the specific intensity $I_{\nu}(s)$ located at $s$ is in units of emitted power per area per photon energy per unit solid angle [which we write as eV $/ \mathrm{sec} / \mathrm{cm}^{2} / \mathrm{eV} / \mathrm{sr}$ ]. The absorption coefficient $\alpha_{\nu}$ is in units of $\mathrm{cm}^{-1}$, and the emission coefficient $\epsilon_{\nu}$ is in units of power per volume per photon energy per unit solid angle [which we write as $\mathrm{eV} / \mathrm{sec} / \mathrm{cm}^{3} / \mathrm{eV} / \mathrm{sr}$ ]. The attenuation experienced by radiation passing through a shell is determined by the optical depth, $\tau_{\nu}=\alpha_{\nu} s$, a dimensionless parameter that describes a materials ability to block radiation. If $\tau_{\nu} \ll 1$, the material is optically thin, and the total intensity is the sum of the emission of each layer. The exponential in Eq. (1) may be expanded as a Taylor series, resulting in the simplified solution

$$
\tau_{\nu} \ll 1: I_{\nu}(s)=I_{\nu}(s=0)+\epsilon_{\nu} s .
$$

If $\tau_{\nu} \gg 1$, the material is optically thick, and the value of the exponential in Eq. (1) is approximately zero, resulting in the simplified solution

$$
\tau_{\nu} \gg 1: I_{\nu}(s)=\frac{\epsilon_{\nu}}{\alpha_{\nu}}
$$

Conceptually, only the emission from the portion of the outermost layer closest to the detector will be observed.

If the plasma is in LTE we have from Kirchhoff's law

$$
\frac{\epsilon_{\nu}}{\alpha_{\nu}}=B_{\nu}(k T)=\frac{2 h \nu^{3}}{c^{2}} /\left(e^{h \nu / k T}-1\right)
$$

where $B_{\nu}(k T)$ is the Planck function, with $k T$ the radiation temperature, $h$ is Planck's constant and $c$ is the speed of light. For non-LTE conditions, the emission and absorption coefficients should be retained explicitly. Planck's function is in the same units as $I_{\nu}$. 


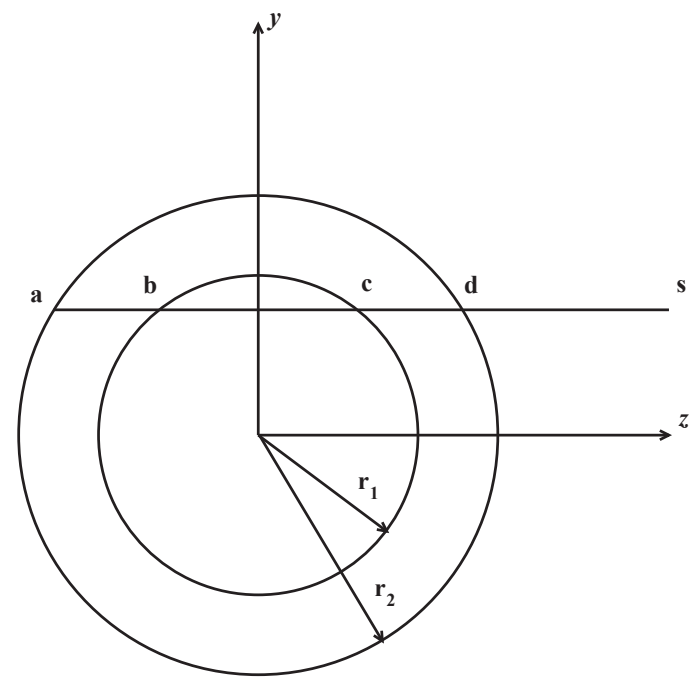

Figure 1: Geometry of the spherical shell model of the emitting plasma. The detector is located on the $z$ axis at some distance $z_{d}>>r_{2}$. A typical chord is given by $s$.

The sum of all the lines of sight passing through a single sphere of radius $r_{2}$ to the detector at $z_{d}$ is then given by the flux

$$
F_{\nu}=2 \pi \int_{0}^{r_{2}} I_{\nu}(L(y)) y d y,
$$

where $L(y)$ is the chord length across the sphere at a given value of $y$. Here the flux $F_{\nu}$ is in units of $\mathrm{eV} / \mathrm{sec} / \mathrm{sr} / \mathrm{eV}$. This is then the total power $/ \mathrm{eV}$ arriving at the location of the detector per unit solid angle (where here we now use photon energy in $\mathrm{eV}$ rather than frequency). For a single uniform sphere the flux can be evaluated analytically ${ }^{43}$. However for the case of concentric shells of differing temperature and/or density the flux must be evaluated numerically. For the case of two shells as seen in figure 1 the radiation must be propagated along the chord from one shell into the next. This is accomplished by applying Eq. 1 first for the chord from $a$ to $b$ with the initial intensity at point $a$ being zero and where $\alpha_{\nu}, \epsilon_{\nu}$ are functions of the density and temperature of the outer shell. This procedure results in 
the intensity at point $b$. The propagation from point $b$ to $c$ is then given by applying Eq. 1 again with the initial value at point $b$ being given by the previous value and $\alpha_{\nu}, \epsilon_{\nu}$ now being functions of the density and temperature of the inner shell. For the intensity at point $d$ we apply Eq. 1 again with the initial value being the final value at point $c$. This yields the final result at point $d$ for a single chord. To evaluate the flux from the sphere we choose chords equally spaced along $y$ with spacing $\Delta y=r_{2} / N$, the number of chords $N$ being chosen such that the numerical integral converges. The above example is easily generalized to any number of concentric spherical zones with the chord lengths being determined by subtraction of the various chord lengths in the individual concentric spheres. If absorption of radiation by the ambient atmosphere is important it can be included by applying Eq. 1 for the chord length from point $d$ to the detector at $z=z_{d}$ with the emission coefficient $\epsilon_{\nu} \approx 0$ and $\alpha_{\nu}$ being that of the ambient atmosphere.

We apply this analysis using a model called FESTR ${ }^{44}$ (Finite-Element Spectroscopic Transport of Radiation), which in addition to spherical geometry will also treat radiation transport through a slab (one line of sight) or a cylinder. Multiple geometries in series can be handled by treating the output of the first geometry as a backlighter on the next, but an external backlighter may also be applied. For each spatial zone (i.e. spherical shell), an electron temperature $(\mathrm{eV})$, mass density $\left(\mathrm{g} / \mathrm{cm}^{3}\right)$, and mass fraction of each element present in the plasma is required. ATOMIC is initialized using these conditions for each zone. The resulting emission and absorption coefficients from each element are then summed to achieve the total emission and absorption coefficients for the plasma. In the case considered here we found that to ensure convergence, a minimum of 10 lines of sight should pass through each shell, with the result that the smallest shell will determine the line of sight spacing for the problem. Once the ATOMIC runs for all shells have been calculated, radiation transport along each line of sight, as presented in figure 1 , can be determined and the flux computed from the spatial integral of the total geometry. Such calculations are presented in Section 4.3.

\section{Modeling of the ChemCam Basalt BIR1 Standard}

\subsection{VNIR Wavelength Region}

We first examine the near-infra-red (NIR) portion of the emission spectrum from the basalt sample BIR1 that is covered by the VNIR ChemCam spectrometer, as was presented previously ${ }^{20}$. The upper panel of figure 2 
shows the ChemCam measurement versus the total emission spectrum from all 13 elements included in the ATOMIC multi-element LTE calculation. The ATOMIC calculation was made at an electron temperature of $0.9 \mathrm{eV}$ (10444 K) and we find very good agreement between the measured spectrum and the ATOMIC total emissivity. The temperature of $0.9 \mathrm{eV}$ was found by best-fit visual comparison of the ChemCam measured spectrum with test calculations at various temperatures, and is found to be reasonably consistent with estimates of the plasma temperatures that were made for the ChemCam observations ${ }^{20}$. We note at this point that in this section we are modeling the emission spectrum assuming a LIBS plasma at a single temperature and density and are not yet including radiation transport effects. Changing the temperature by $\pm 0.2 \mathrm{eV}$ resulted in worse agreement between the ATOMIC calculations and measured spectrum for the line ratios of the prominent emission features in this wavelength range. The ATOMIC calculations were made for the 13 elements at the mass densities listed in Table 3. We note again that this calculation includes an arbitrary amount of $\mathrm{H}$ (from possible sample hydration), $\mathrm{C}$ (from atmospheric $\mathrm{CO}_{2}$ coupling to the plasma) and increased amounts of $\mathrm{O}$ that may arise from both hydration and atmospheric effects. The global electron density that results from this calculation is $2.7 \times 10^{16} \mathrm{~cm}^{-3}$ as computed by ATOMIC. In the lower panel of figure 2 we show the contributions from each individual element in the ATOMIC calculation. We note the strong $\mathrm{O}$ emission lines at $777 \mathrm{~nm}$ (this feature is really a triplet of lines that appear as a single emission peak due to spectral resolution), as well as weaker $\mathrm{O}$ features at around $845 \mathrm{~nm}$. Other prominent features are the $\mathrm{Mg}$ I lines near $517 \mathrm{~nm}$ and the $\mathrm{Na} I$ lines near $589 \mathrm{~nm}$ and $820 \mathrm{~nm}$. The latter emission feature (at $820 \mathrm{~nm}$ ), which is the result of an excited-state to excited-state transition $(3 d \rightarrow 3 p)$, shows some disagreement in emission intensity between the ATOMIC calculation and the measurement. Other notable features of this spectrum are the $\mathrm{Ca}$ lines at various wavelength between $520 \mathrm{~nm}$ and $720 \mathrm{~nm}$, and a Si II emission line near $634 \mathrm{~nm}$. Figure 3 shows zoomed in regions of this spectrum that also identifies the more prominent individual lines. The middle panel of figure 2 shows a residual between the experimental data and the ATOMIC calculation, simply defined as the difference between the experimental and theoretical data divided by the theoretical data. The largest differences are observed around $500 \mathrm{~nm}$, where the calculation under-predicts the measured emission, and at around $589 \mathrm{~nm}$, where small differences in the line shapes of the measured and calculated spectrum contribute to a substantial difference 
in the resulting residual.

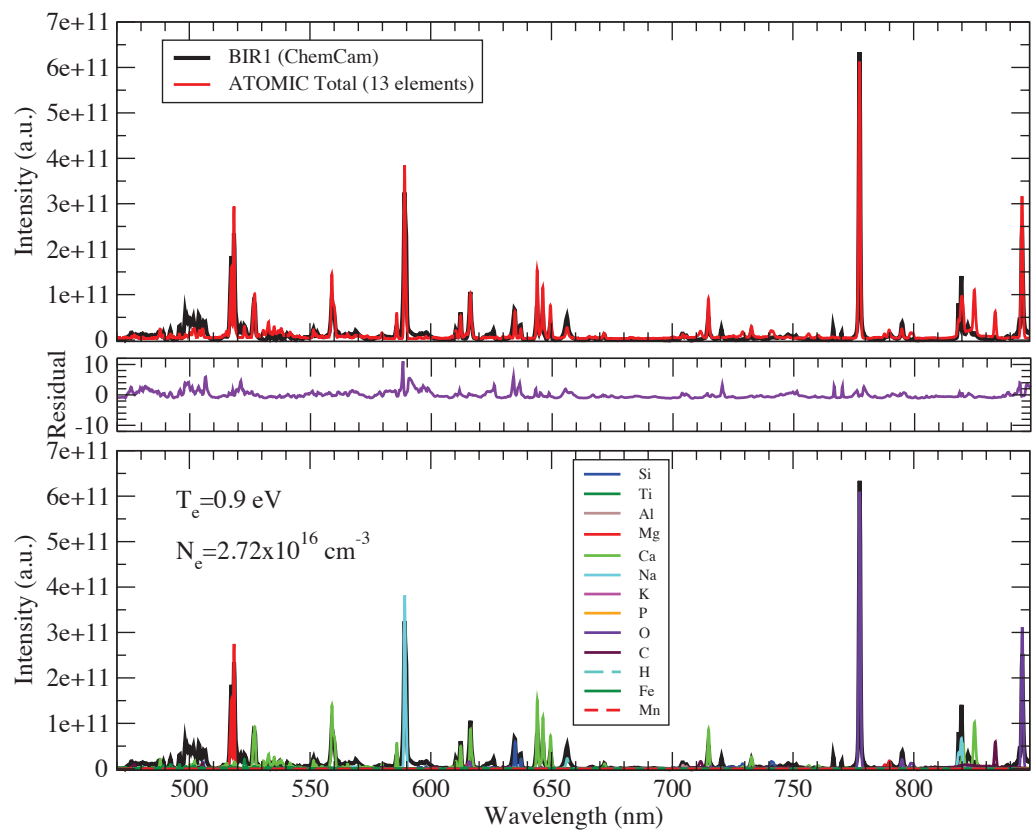

Figure 2: Comparison of the ChemCam spectra from the basalt sample BIR1 with the ATOMIC multi-element LTE calculation at a temperature of $0.9 \mathrm{eV}$ and for a wavelength range of 470 to $850 \mathrm{~nm}$. The upper panel shows the total emissivity from ATOMIC (red line) versus the ChemCam basalt BIR1 standard. The lower panel shows the contributions from the different elements present in the basalt plasma. The global electron density as computed from ATOMIC is also indicated. The middle panel shows a residual between the experimental data and the ATOMIC calculations as described in the text.

To underscore the need for a true multi-element approach to determining level populations in the modeling of the basalt plasma, in figure 4 we present emission spectra from basalt at the same conditions as in figure 2, except that each elemental spectra is computed from a single-element calculation, each at the electron density that is found from a single-element calculation at a temperature of $0.9 \mathrm{eV}$ and the mass density listed in table 3 . We find much poorer agreement between the ChemCam spectrum and the ATOMIC calculations, with almost all of the emission lines found to be much weaker than in the multi-element calculations. Although one could consider emission from the plasma constituents at higher mass densities (resulting in higher elec- 


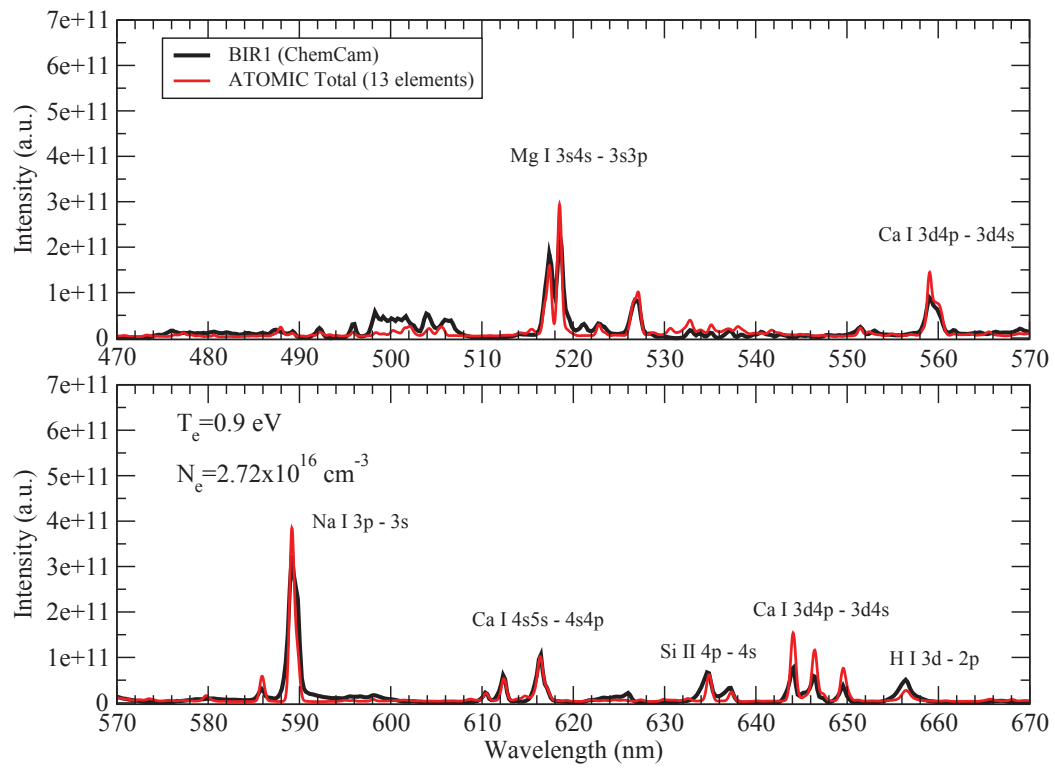

Figure 3: Comparison of the ChemCam spectra from the basalt sample BIR1 with the ATOMIC multi-element LTE calculation at a temperature of $0.9 \mathrm{eV}$ and in the wavelength range of 470 to $570 \mathrm{~nm}$ (upper panel) and 570 to $670 \mathrm{~nm}$ (lower panel).

tron densities) that would increase the intensity of the emission lines, such calculations would no longer reflect the elemental abundances of basalt. We find that the electron density computed for each separate calculation is quite different than the electron density found in the multi-element calculation. For example, the electron density found in the single-element Na calculation is $3.5 \times 10^{14} \mathrm{~cm}^{-3}$ and for the single-element Ca calculation the electron density was found to be $1.4 \times 10^{14} \mathrm{~cm}^{-3}$, both of which are much smaller than the global electron density of $2.7 \times 10^{16} \mathrm{~cm}^{-3}$ found in the multi-element calculation. We observe that only the $\mathrm{O}$ lines are still prominent in the single-element calculations shown in figure 5. The poor agreement between the measured spectrum and single-element ATOMIC calculations is also reflected in the residual, shown in the lower panel of figure 4 . The residual generally is much larger for the single-element case compared to the multielement calculation shown in figure 2. The large difference in the electron density found in the multi-element ATOMIC calculations compared to the 
electron density found in the single-element ATOMIC calculations appears to be the origin of some of the 'matrix effects' phenomenon that has been often referred to in the literature. The higher electron density found in a unified treatment of all the plasma constituents can significantly alter the intensity of the emission lines from any one species compared to consideration of each plasma component separately. The wide range of electron densities found in the single element calculations also shows how a multi-element treatment, with an often larger electron density, can drive the system further into LTE.

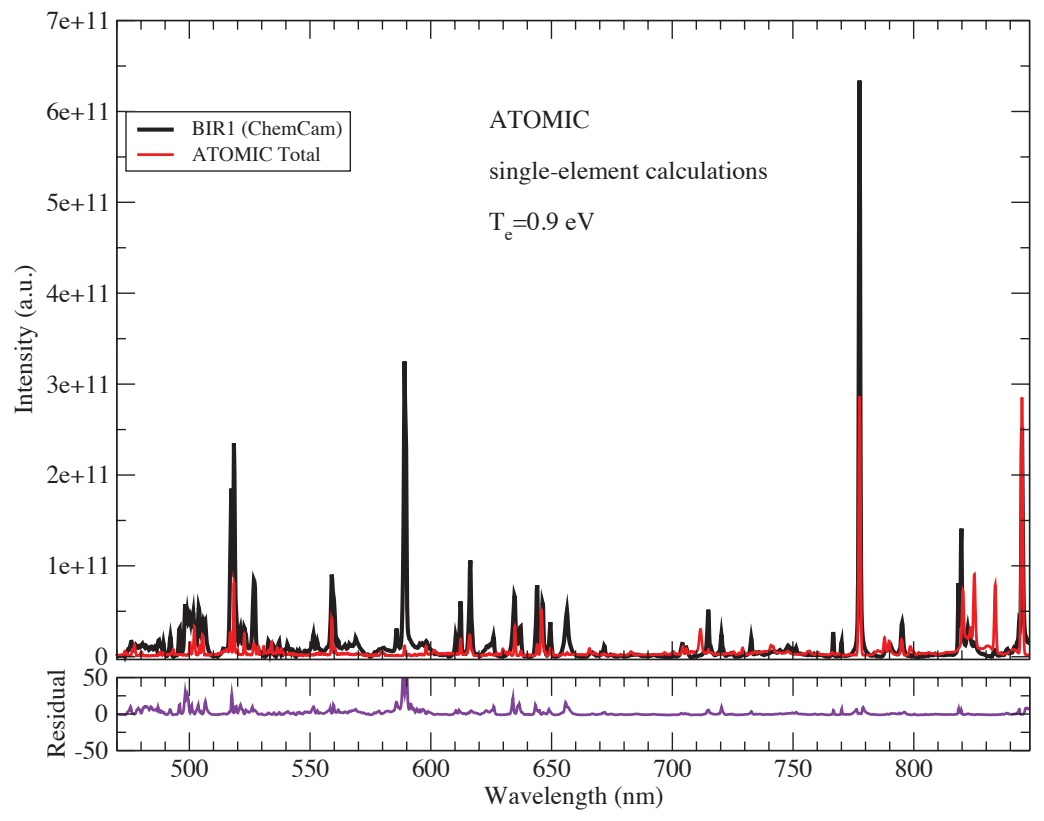

Figure 4: Comparison of the ChemCam spectra from the basalt sample BIR1 with a set of single-element ATOMIC calculations for the same plasma compositions as in figure 4, and at an electron temperature of $0.9 \mathrm{eV}$. The lower panel shows a residual between the experimental data and the ATOMIC calculations as described in the text.

It is also instructive to consider non-LTE effects in this plasma. Figure 5 shows a non-LTE calculation (made by setting the radiation temperature to zero) from ATOMIC. Unfortunately, the much greater computational resources required for non-LTE calculations did not allow us to perform the non-LTE calculation using exactly the same model as for the LTE calculations presented in figures 2 and 3. We were forced to omit the Fe and $\mathrm{Mn}$ 
species from our non-LTE calculations (due to the large size of the atomic models for these elements) so that a tractable non-LTE calculation could be performed. We note that Fe and Mn have almost no prominent emission lines in this wavelength region. In figure 5 we show in the upper panel the emissivity from such a non-LTE calculation. The lower panel shows the LTE calculation for exactly the same conditions. We find that the non-LTE calculation is quite similar to the LTE calculation, and the electron density produced from the non-LTE calculation is around 5\% lower than the electron density from the LTE calculation. The spectral features are almost unchanged, with only small changes in some line heights found. This implies that the plasma created from the basalt target is close to LTE, which is consistent with our earlier study of the electron densities at which LTE is reached in such plasmas ${ }^{31}$.

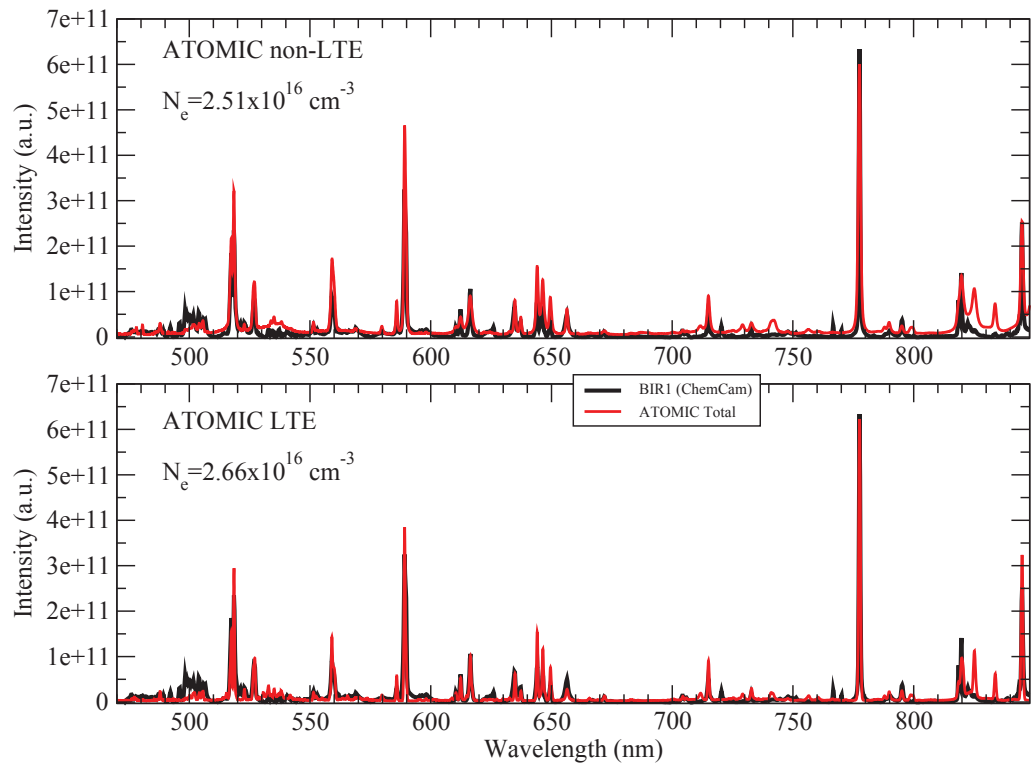

Figure 5: Comparison of the ChemCam spectra from the basalt sample BIR1 with the ATOMIC non-LTE (upper panel) and LTE calculations (lower panels) for the same plasma composition as used for the calculations in figure 2, except that Fe and $\mathrm{Mn}$ have been omitted from the calculations. 


\subsection{UV/VIO Wavelength Region}

We now examine the spectrum of basalt in the wavelength region covered by the UV and VIO ChemCam spectrometers, that is from $240 \mathrm{~nm}$ to $483 \mathrm{~nm}$. Figure 6 shows the ATOMIC LTE multi-element calculation at an electron temperature of $0.9 \mathrm{eV}$, where again the upper panel shows the total emissivity, and the lower panel shows the contributions from each element. We find that the agreement in this wavelength region is much poorer than found for the VNIR region discussed in the previous section. In particular, the ATOMIC calculations significantly over-predict (by around a factor of 20) the Mg II emission lines around $279 \mathrm{~nm}$ and the Ca II emission lines at 393 and $397 \mathrm{~nm}$. We also find that although ATOMIC does a reasonably good job of predicting the position of the closely-spaced Fe lines (around $260 \mathrm{~nm}$ ) and Ti lines (around $323 \mathrm{~nm}$ ), the intensities of these lines are in only fair agreement with the ChemCam measurement.

There are several reasons for the discrepancy between the ATOMIC modeling and the ChemCam spectrum in this range. The strong Mg II lines at $279 \mathrm{~nm}$ are the $3 p$ to $3 s$ transitions, and the strong Ca II lines at 393 and $397 \mathrm{~nm}$ are the $4 p$ to $4 s$ transitions. The Ca II line at $397 \mathrm{~nm}$ appears to be saturated in the ChemCam measurement, so that it's true height would be somewhat larger than recorded by the ChemCam instrument. More importantly, however, these sets of lines are not optically thin, and so the emission at these wavelengths will be modified by the radiation transport through the plasma. This effect can be estimated by examining the optical depth $(\tau)$ as a function of wavelength in this region. The optical depth is shown in figure 7, for a representative plasma length of $750 \mu \mathrm{m}$ (which is a reasonable plasma size $^{20}$, discussed further below). The optical depth is computed by calculating the absorption coefficient, $\alpha_{\nu}$, of the basalt sample and multiplying by the plasma length. We see that the optical depth for the Mg II and Ca II lines is of order 1.0, indicating that these lines will in fact suffer absorption as the radiation is transported through the plasma.

\subsection{Radiation Transport Calculations}

To address the issue of emission lines that are not optically thin as discussed in the previous section, we performed some calculations using the radiation transport model discussed in Section 3.1 using the FESTR code ${ }^{44}$. We explored the sensitivity of the emission spectrum to the number of zones of different temperatures and densities as well as the radial extent of such zones. We also checked convergence of the number of lines of sight included in 


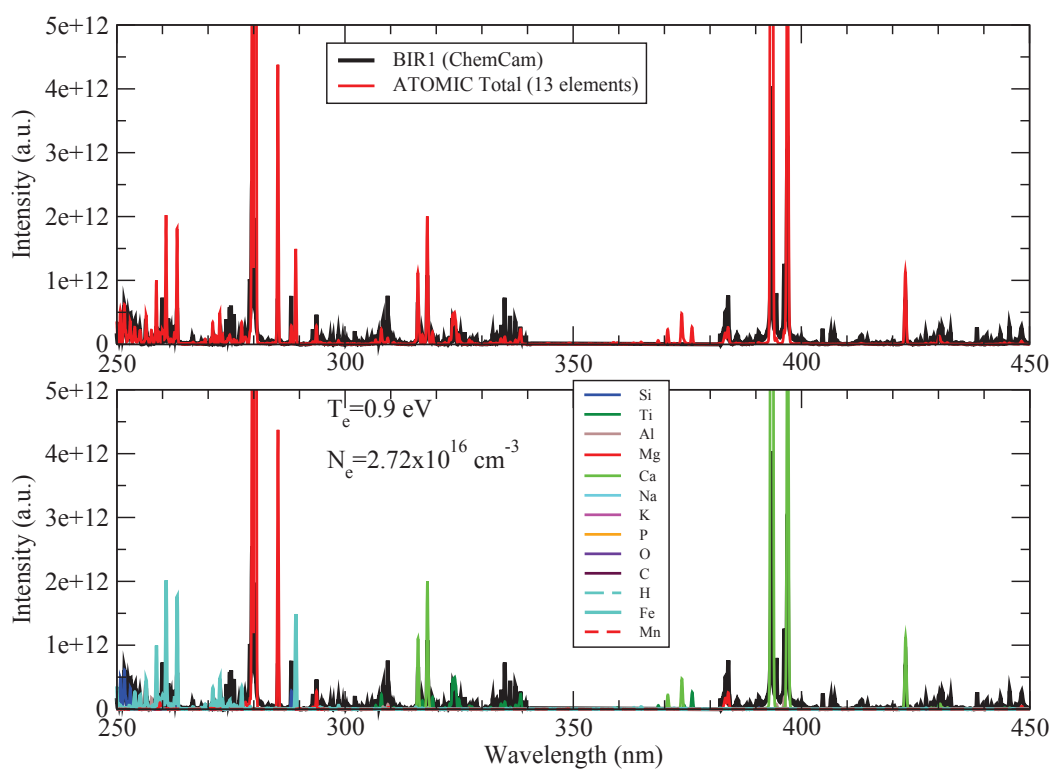

Figure 6: Comparison of the ChemCam spectra from the UV and VIO spectrometers from the basalt sample BIR1 with the ATOMIC multi-element LTE calculation at a temperature of $0.9 \mathrm{eV}$ and for a wavelength range of 250 to $450 \mathrm{~nm}$. The upper panel shows the total emissivity from ATOMIC (red line) versus the ChemCam spectrum. The lower panel shows the contributions from the different elements present in the basalt plasma. The global electron density as computed from ATOMIC is also indicated.

the radiation transport calculation. After such studies, we arrived at a tentative radiation transport model that appears to be physically plausible. Our model uses 101 lines of sight through the plasma and consists of 3 distinct plasma zones at different temperatures and densities with each zone having a different radial extent. Our picture of the LIBS plasma is of an inner zone at a temperature of $1.2 \mathrm{eV}$ and at a density of 5 times the mass densities quoted in Table 3. The central zone is modeled at a temperature of $0.9 \mathrm{eV}$ and at mass densities quoted in Table 3 and the outer zone is at a temperature of $0.6 \mathrm{eV}$ and at mass densities 0.2 times the mass densities quoted in Table 3. The zone radii are $700 \mu \mathrm{m}, 850 \mu \mathrm{m}$, and $1000 \mu \mathrm{m}$, where these values were obtained by testing the sensitivity of the attenuation of the major emission lines to the radii of each zone. Thus our scenario is of a plasma that is hot- 


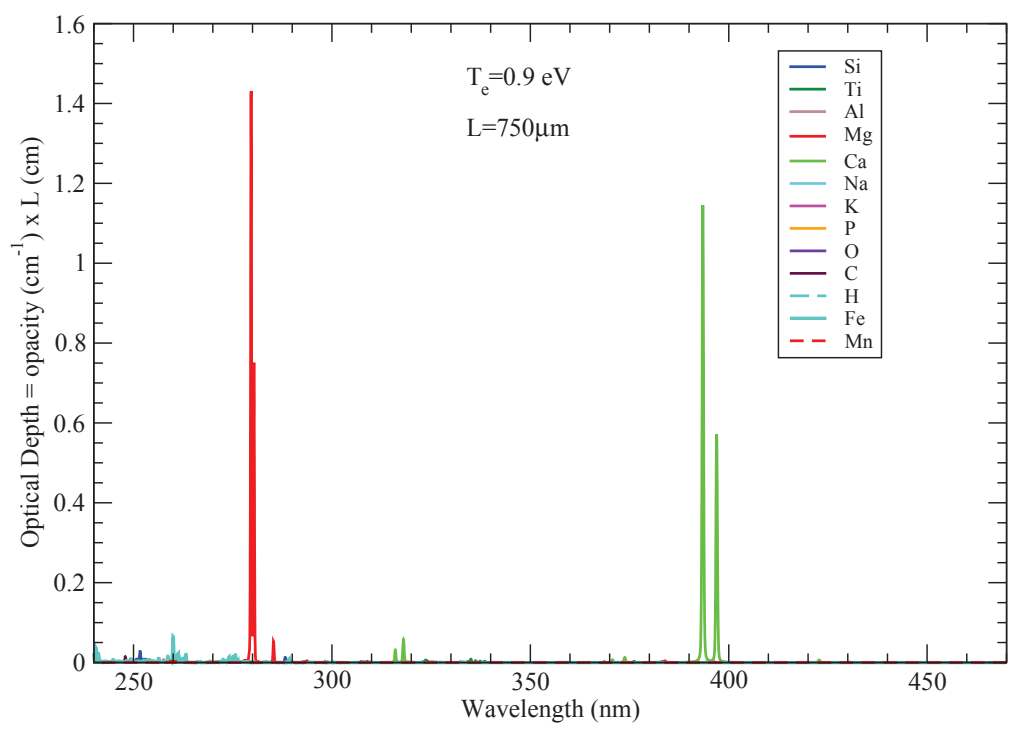

Figure 7: Optical depth of the basalt sample in the UV/VIO wavelength region as computed from the ATOMIC calculations described in the text. The electron temperature is $0.9 \mathrm{eV}$ and we assume a plasma length of $750 \mu \mathrm{m}$.

ter and denser at the center, and has outer layers that are cooler and less dense-a model that is consistent with a volumetrically expanding plasma ${ }^{45}$. We also tested if the addition of a cooler, atmospheric layer of $\mathrm{CO}_{2}$ resulted in any further attenuation of the observed emission and found that such a layer had a negligible effect. The results of these radiation transport calculations are shown in figure 8 for the UV/VIO wavelength range. We have utilized a least squares model ${ }^{46}$ to reconcile the radiation transport model units to the arbitrary units of the data. The scaling factor to be applied to each radiation transport solution is determined though minimization of the $\chi^{2}$ for each fit such that the baseline of the model solution and experimental data agree. We have found using this process that changes of $\pm 50 \mu \mathrm{m}$ in any zone radii will reduce the level of agreement between the model and the data. It should be noted that the zone radii described here correspond to a plasma thickness, i.e. the depth of plasma through which light must travel to reach the observer. We find that the resonance Mg II and Ca II lines 
are significantly attenuated (by more than a factor of ten) by the transport through the various plasma zones, whereas the other, weaker, emission lines are much less affected by the transport. Including the radiation transport thus brings the ATOMIC calculations into much better agreement with the ChemCam spectrum in this wavelength region, as is further illustrated by the zoomed in region presented in figure 9. We note that the attenuation of the resonance lines in Mg II and Ca II is primarily a self-absorption process. In other words, it is not that the hot inner layer emits these lines exclusively, and a cooler outer layer is opaque to them, as is expressed by the first term of Eq. (1), but that the opacity of the very ions which produce these emission lines results in less emission exiting the inner layer in the first place, as in the 2nd term of Eq. (1). The $0.9 \mathrm{eV}$ and $0.6 \mathrm{eV}$ layers are necessary not because they attenuate these resonance lines, but because they add other emission lines observed in the experimental data that come from states which do not have sufficient population at $1.2 \mathrm{eV}$ to produce significant emission.

We note that the emission in the VNIR wavelength region that was explored in Section 4.1 is generally much less intense than the emission in the shorter-wavelength near-visible region. The emission in the VNIR region is optically thin and exhibits little sensitivity to radiation transport. However, using the 3 zone plasma conditions that work well for the UV/VIO region for the VNIR wavelength region results in a comparison (not shown) between the calculations and the ChemCam measurement that is slightly worse than that shown in figures 2 and 3. In particular, the Mg I and Na I lines (near $518 \mathrm{~nm}$ and $589 \mathrm{~nm}$ respectively) are at a lower intensity in the calculations compared to the measurement, due to the influence of the hotter central zone. This implies an inconsistency in our modeling of the different wavelength regions - a 3 zone model works well in one wavelength region, but does not work quite as well (compared to a single zone calculation) in another wavelength region. This finding is likely due to our incomplete picture of the plasma physics processes underway. Although we have presented calculations that reveal the importance of multi-element effects and of radiation transport, a comprehensive understanding of the LIBS plasma is still difficult to attain. For example, perhaps the spatial variation in temperature and/or density of the LIBS plasma is such that two-dimensional radiation transport is required (not 1-D as is assumed in the calculations presented here). However, to perform more sophisticated radiation transport modeling would require detailed knowledge about the temperature and density profile of the LIBS-generated plasma-a task that requires radiation-hydrodynamics calculations. We hope 
to perform such calculations in future work. We have also not considered any possible temporal variation in the plasma emission or any potential shot-toshot variation in the ChemCam data that might affect the quality of the comparison between the archived measurements and the calculations.

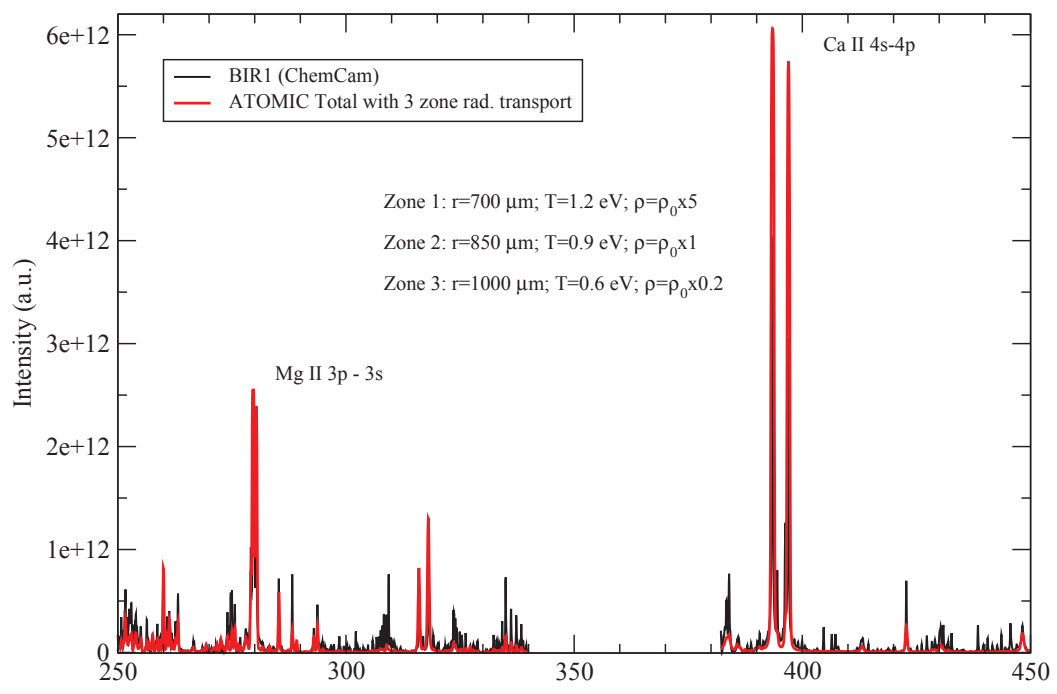

Figure 8: Emission spectrum of the Basalt sample in the near-visible region as computed from the ATOMIC calculations that include radiation transport through three plasma zones as described in the text. Each zone radius is indicated as well as the temperature in each zone. The densities used in each zone are factors of $\rho_{0}$, where $\rho_{0}$ represents the mass densities of all the elements listed in Table 3.

\section{High-Resolution Measurements of Basalt}

To complete our investigation of the emission spectrum from basalt, we also employed a high-resolution spectrometer to capture the emission spectrum from the same basalt sample (BIR1) as used in the ChemCam spectra. This study was aimed at exploring in more detail various wavelength ranges of the basalt emission spectrum so that finer details of the emission spectrum may be studied. Although the majority of the emission features measured 


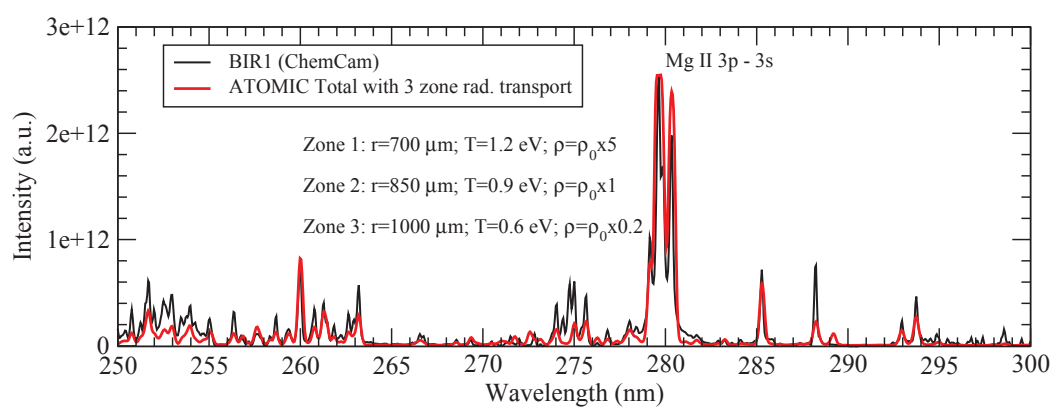

Figure 9: Emission spectrum of the Basalt sample in the $250-300 \mathrm{~nm}$ region as computed from the ATOMIC calculations that include radiation transport through three plasma zones as described in the text.

in the high-resolution measurements matched the ChemCam standard measurements very well, some differences were observed. In particular, the $\mathrm{O}$ $777 \mathrm{~nm}$ emission line in the newer measurements was not as intense as found in the ChemCam spectra and also the weaker H $656 \mathrm{~nm}$ line was almost absent in the newer measurements. This may be because the BIR1 sample was more hydrated in the original ChemCam measurement than in the newer measurements, which is plausible as the samples used to build the ChemCam standards database are not generally stored in a climate-controlled environment. We also found some sensitivity to the time-gating of the newer measurements. The best match between the new high-resolution measurements and the ChemCam spectra was found when no gating was employed in the new measurements (the ChemCam instrument has no timing controls and so all emission is captured). In the new measurements, when various gating widths and delays are employed, some emission features were found to be sensitive to the gate settings. Such variation will be the subject of a future study.

In figure 10 we present the $515-530 \mathrm{~nm}$ wavelength portion of the BIR1 emission spectrum. The upper panel shows the emission collected using the ChemCam VNIR spectrometer and the lower panel shows the emission measured using the high-resolution spectrometer. In each panel, we compare to ATOMIC calculations made at the same conditions as shown in figures 2 and 3. The only difference in the two calculations is the convolution with the resolution of each spectrometer; a resolving power of $\sim 1000$ was used in 
the calculations shown in the upper panel and a resolving power of $\sim 10000$ was used in the lower panel. We find that, as expected, the high-resolution measurements show significantly more detail. The Mg I lines around $517 \mathrm{~nm}$ are now clearly resolved into 3 components, and the Ca I emission feature at $527 \mathrm{~nm}$, which is a single line in the ChemCam spectrometer, is now resolved into 4 lines in the high-resolution measurement.

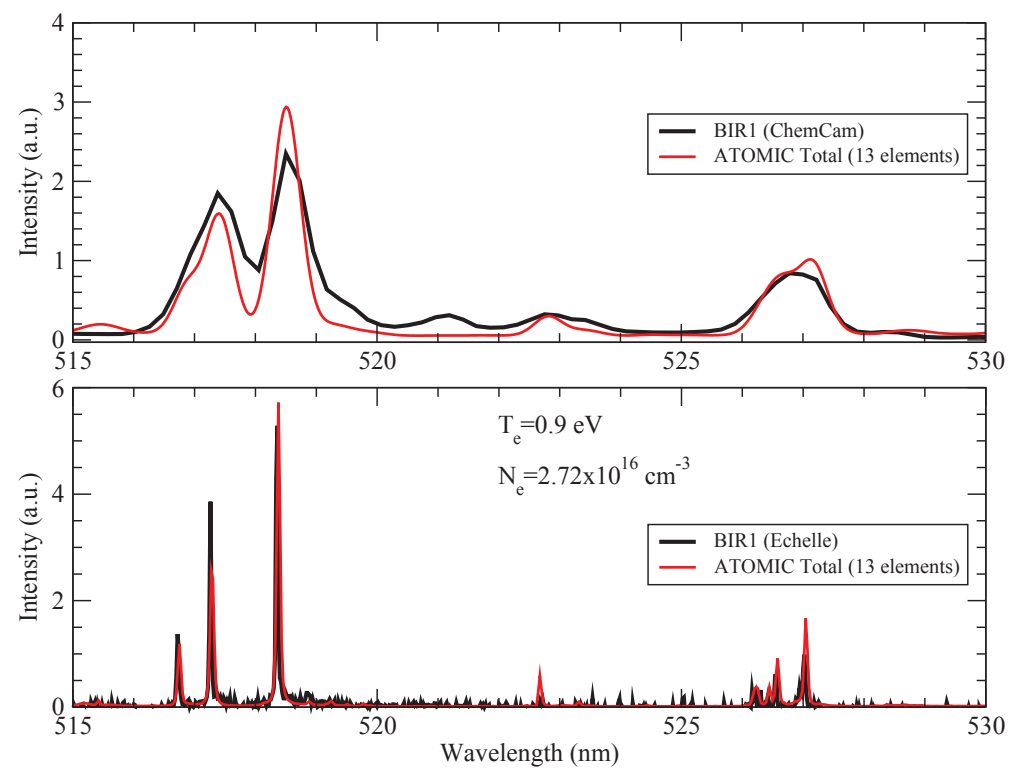

Figure 10: Emission spectrum of the basalt sample in the 515-530 $\mathrm{nm}$ wavelength region. Upper panel: basalt spectrum (black line) measured using the ChemCam spectrometer and compared to an ATOMIC multi-element calculation (red line) at a temperature of $0.9 \mathrm{eV}$ and the mass densities listed in Table 3. Lower panel: basalt spectrum measured using the high-resolution Echelle spectrometer and compared to an ATOMIC calculation performed at the same conditions as the ATOMIC calculations in the upper panel. The resolution of each spectrometer is included in the ATOMIC calculation in each case.

In figure 11, we present another portion of the basalt emission spectrum in the 315-330 nm wavelength range. A similar comparison to the one shown in figure 9 is made, except that the ATOMIC calculations shown here are from the 3-zone radiation transport calculations shown in figure 8 . We again find that the high-resolution measurements yield significantly more detail of 


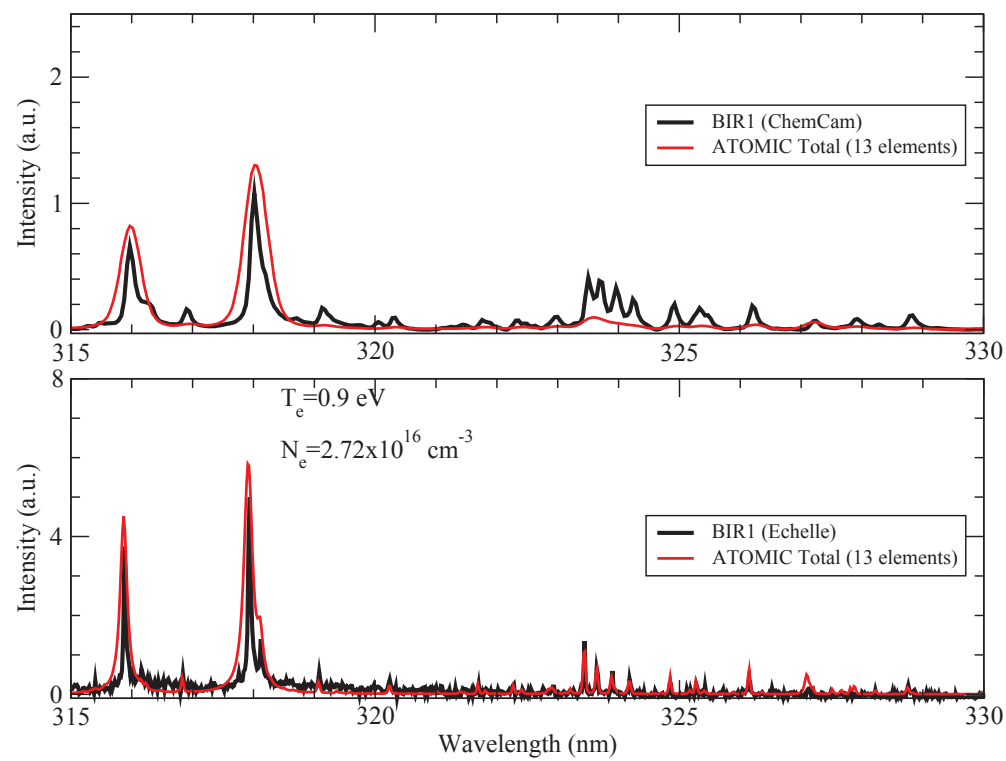

Figure 11: Emission spectrum of the basalt sample in the 315-330 $\mathrm{nm}$ wavelength region. Upper panel: basalt spectrum (black line) measured using the ChemCam spectrometer and compared to an ATOMIC multi-element calculation (red line) at the same conditions as used for the calculations shown in figure 8. Lower panel: basalt spectrum measured using the high-resolution Echelle spectrometer and compared to an ATOMIC calculation performed at the same conditions as the ATOMIC calculations in the upper panel. The resolution of each spectrometer is included in the ATOMIC calculation in each case.

the emission spectrum. The Ti emission lines around $323 \mathrm{~nm}$ are now well resolved, as are the Ca II 4d-4p lines at 315 and $318 \mathrm{~nm}$.

\section{Conclusions}

In this paper we have analyzed the emission spectrum from basalt, a geologically common rock that was used as one of the calibration standards for the ChemCam instrument on the Mars Science Laboratory. The Los Alamos ATOMIC code was used to construct emission spectrum for all the major elements present in basalt. Our study demonstrates that quantitative modeling of complex materials containing many elements is possible and reveals various aspects of the physics of LIBS plasmas. For example, we 
have shown that one must consider a multi-element plasma picture where all elements are affected by a global electron density that is constructed from the partial ionization of all species within the plasma. The influence of such a global electron density appears to be the origin of some of the 'matrix effects' often discussed in the LIBS literature, where the emission spectrum from one element is influenced by the presence of other species within the plasma.

We have also demonstrated that radiation transport through the LIBS plasma can be important and may significantly alter the intensity of strong emission lines observed in a LIBS plasma emission spectrum. We have used ATOMIC in conjunction with the FESTR code to construct a simple radiation transport model that shows how strong resonance lines from Ca II and $\mathrm{Mg}$ II ions are strongly attenuated by transport through the plasma. The temperatures and densities of the radiation transport zones used in this present work are consistent with a volumetrically expanding plasma. Changes in temperatures of more then $\pm 0.2 \mathrm{eV}$ are enough to significantly alter the ionization balance and the emission of the plasma and result in poorer agreement with the measured spectra. The plasma properties are however less sensitive to variations in density. In the future, we plan further development of radiation hydrodynamic calculations that may predict the temperature and density temporal and spatial profile of a LIBS plasma. Such calculations would allow us a more complete picture of the evolving plasma dynamics of the system, and allow more accurate calculations of the detected emission.

\section{Acknowledgments}

The Los Alamos National Laboratory is operated by Los Alamos National Security, LLC for the NNSA of the U.S. DOE under Contract No. DE-AC5206NA25396. This work was carried out under laboratory-directed research and development funding.

\section{References}

[1] L.J. Radziemski, D. Cremers, Laser-Induced Plasmas and Applications, (Marcel Dekker, Inc, New York, 1989).

[2] L.J. Radziemski, From LASER to LIBS, the path of technology development, Spectrochimica Acta B 57 (2002) 1109-1113. 
[3] M Capitelli, A. Casavola, G. Colonna, A. De Giacomo, Laser-induced plasma expansion: theoretical and experimental aspects, Spectrochimica Acta Part B 59 (2004) 271-289.

[4] R. Noll, Laser-Induced Breakdown Spectroscopy, (Springer-Verlag, Heidelberg 2012).

[5] G. Cristoforetti, E. Tognoni, L.A. Gizzi, Thermodynamic equilibrium states in laser-induced plasmas: From the general case to laser-induced breakdown spectroscopy plasmas, Spectrochimica Acta B 90 (2013) 122 .

[6] L. Radziemski, D. Cremers, A brief history of laser-induced breakdown spectroscopy: From the concept of atoms to LIBS 2012, Spectrochimica Acta B 87 (2013) 3-10.

[7] J. Vrenegor, R. Noll, V. Sturm, Investigation of matrix effects in laserinduced breakdown spectroscopy plasmas of high-alloy steel for matrix and minor elements, Spectrochimica Acta B 60 (2005) 1083-1091.

[8] Y. Iida, Effects of atmosphere on laser vaporization and excitation processes in solid samples, Spectrochimica Acta B 45 (1990) 1353-1367.

[9] C.A. Henry, P.K. Diwakar, D.W. Hahn, Investigation of helium addition from laser-induced plasma spectroscopy of pure gas phase systems: Analyte interactions and signal enhancement, Spectrochimica Acta B 62 (2007) 1390-1398.

[10] P.K. Diwakar, P.B. Jackson, D.W. Hahn, The effect of multi-component aerosol particles on quantitative laser-induced breakdown spectroscopy: Consideration of localized matrix effects, Spectrochimica Acta B 62 (2007) 1466-1474.

[11] E. Tognoni, M. Hidalgo, A. Canals, G. Cristoforetti, S. Legnaioli, V. Palleschi, Combination of the ionic-to-atomic line intensity ratios from two test elements for the diagnostic of plasma temperature and electron number density in Inductively Coupled Plasma Atomic Emission Spectroscopy, Spectrochimica Acta B 62 (2007) 435-443.

[12] J.A. Aguilera, C. Aragón, V. Madurga, J. Manrique, Study of matrix effects in laser induced breakdown spectroscopy on metallic samples using 
plasma characterization by emission spectroscopy, Spectrochimica Acta B 64 (2009) 993-998.

[13] M. Dong, X. Mao, J.J. Gonzalez, J. Lu, R.E. Russo, Time-resolved LIBS of atomic and molecular carbon from coal in air, argon and helium, J. Anal. At. Spectrom. 27 (2012) 2066-2075.

[14] http://mars.jpl.nasa.gov/msl/

[15] R.C. Wiens, S. Maurice, and the MSL Science Team (2015) ChemCam: Chemostratigraphy by the first Mars microprobe. Elements, 11, 33-38.

[16] C. Fabre, S. Maurice, A. Cousin, R.C. Wiens, O. Forni, V. Sautter, D. Guillaume, Onboard calibration igneous targets for the Mars Science Laboratory Curiosity rover and the Chemistry Camera laser induced spectroscopy instrument, Spectrochimica Acta B 66 (2011) 280-289.

[17] D. Vaniman, M.D. Dyar, R. Wiens, A. Ollila, N. Lanza, J. Lasue, J.M. Rhodes, S. Clegg, H. Newsom, Ceramic ChemCam calibration targets on Mars Science Laboratory, Space. Sci. Rev. 170 (2012) 229-255.

[18] M.D. Dyar, J.M. Tucker, S. Humphries, S.M. Clegg, R.C. Wiens, M.D. Lane, Strategies for Mars remote laser-induced breakdown spectroscopy analysis of sulfur in geological samples, Spectrochimica Acta B 66 (2011) $39-56$.

[19] O. Forni, S. Maurice, O. Gasnault, R.C. Wiens, A. Cousin, S.M. Clegg, J.-B. Sirven, J. Lasue, Independent component analysis classification of laser induced breakdown spectroscopy spectra, Spectrochimica Acta B 86 (2013) 31-41.

[20] R.C. Wiens, S. Maurice, J. Lasue, O. Forni, R.B. Anderson, S. Clegg, S. Bender, D. Blaney, B.L. Barraclough, A. Cousin, L. Deflores, D. Delapp, M.D. Dyar, C. Fabre, O. Gasnault, N. Lanza, J. Mazoyer, N. Melikechi, P.-Y. Meslin, H. Newsom, A. Ollila, R. Perez, R.L. Tokar, D. Vaniman, Pre-flight calibration and initial data processing for the ChemCam laser-induced breakdown spectroscopy instrument on the Mars Science Laboratory rover, Spectrochimica Acta B 82 (2013) 1-27. 
[21] S. Maurice, R.C. Wiens, M. Saccoccio, B. Barraclough, O. Gasnault, O. Forni, et al., The ChemCam instruments on the Mars Science Laboratory (MSL) rover: science objectives and mast unit, Space Sci. Rev., 170 (2012), pp. 95-166.

[22] R. Wester, R. Noll, Heuristic modeling of spectral plasma emission for laser-induced breakdown spectroscopy, J. Applied Phys. 106 (2009) 123302 .

[23] A.R Casavola, G. Colonna, M. Capitelli, Kinetic model of titanium laser induced plasma expansion in nitrogen environment, Plasma Sources Sci. Technol. 18 (2009) 025027.

[24] S.T. Surzhikov, M. Capitelli, G. Colonna, C. Gorse, Radiative gas dynamic model of hydrogen laser-supported plasma generator, J. Thermophysics and Heat Transfer 22 (2008) 62-70.

[25] L.D. Pietanza, G. Colonna, A. De Giacomo, M. Capitelli, Kinetic processes for laser induced plasma diagnostic: A collisional-radiative model approach, Spectrochimica Acta B 65 (2010) 616-626.

[26] P.K. Diwakar, D.W. Hahn, Study of early laser-induced plasma dynamics: Transient electron density gradients via Thomson scattering and stark broadening, and the implications on laser-induced breakdown spectroscopy measurements, Spectrochimica Acta B 63 (2008) 1038-1046.

[27] I.B. Gornushkin, A.Ya. Kazakov, N. Omenetto, B.W. Smith, J.D. Winefordner, Radiation dynamics of post-breakdown laser induced plasma, Spectrochimica Acta B 59 (2004) 401-418.

[28] S.S. Harilal, G.V. Miloshevsky, P.K. Diwakar, N.L. LaHaye, A. Hassanein, Experimental and computational study of complex shockwave dynamics in laser ablation plumes in argon atmosphere, Physics of Plasmas 19 (2012) 083504.

[29] A.E. Hussein, P.K. Diwakar, S.S. Harilal, A. Hassanein, The role of laser wavelength on plasma generation and expansion of ablation plumes in air, Journal of Applied Physics 113 (2013) 143305.

[30] N.H. Magee, J. Abdallah, J. Colgan, P. Hakel, D.P. Kilcrease, S. Mazevet, M. Sherrill, C.J. Fontes, H.L. Zhang, Los Alamos Opacities: 
Transition from LEDCOP to ATOMIC, 14th Topical Conference on Atomic Processes in Plasmas, (Eds: J.S. Cohen, S. Mazevet, D.P. Kilcrease, AIP Conference Proceedings, New York, 2004) 168-179.

[31] J. Colgan, E.J. Judge, D.P. Kilcrease, J.E. Barefield II, Ab-initio modeling of an iron laser-induced plasma: Comparison between theoretical and experimental atomic emission spectra, Spectrochimica Acta B 97 (2014) 65-73.

[32] R.D. Cowan, The Theory of Atomic Structure and Spectra, (University of California Press, Berkeley, 1981).

[33] J. Abdallah, R.E.H. Clark, R.D. Cowan, CATS: Cowan atomic structure code, Los Alamos National Laboratory, Los Alamos Manual No. LA 11436-M-I (1988).

[34] http://physics.nist.gov/PhysRefData/ASD/

[35] R.E.H. Clark, J. Abdallah Jr, J.B. Mann, Integral and differential cross sections for electron impact ionization, Ap. J. 381 (1991) 597-600.

[36] E.J. Judge, G. Peterson, R. Newell, et al., in preparation (2015).

[37] M.E. Sherrill, R.C. Mancini, J. Bailey, A. Filuk, B. Clark, P. Lake, J. Abdallah, Jr., Spectroscopic modeling and characterization of a collisionally confined laser-ablated plasma plume, Phys. Rev. A 76 (2007) 056401.

[38] A.N. Cox, J.N. Stewart, D.D. Eilers, Effects of bound-bound absorption on stellar opacities, Ap. J. S 11 (1965) 1.

[39] A.N. Cox, Stars and Stellar Systems, Vol 8, (Chicago: University of Chicago Press, 1964).

[40] W.F. Huebner, W.D. Barfield, Opacity, (Springer, New York, 2014).

[41] M.S. Dimitrijević and N. Konjević, Simple formulae for estimating Stark widths and shifts of neutral atom lines, Astron. Astrophys. 163 (1986) 297-300.

[42] D. Mihalas, Stellar Atmospheres, 2nd ed. (Freeman, 1978). 
[43] T.J. Burris-Mog, R.C. Mancini, J.E. Bailey, G.A. Chandler, G. Rochau, G. Dunham, P.W. Lake, K. Peterson, S.A. Slutz, T.A. Mehlhorn, I.E. Golovkin, J.J. MacFarlane, Line broadening analysis of implosion core conditions at Z using argon K-shell spectroscopy, J. Quant. Spectrosc. Radiat. Transfer 99 (2006) 120-130.

[44] P. Hakel, G.A. Kyrala, P.A. Bradley, N.S. Krasheninnikova, T.J. Murphy, M.J. Schmitt, I.L. Trigillis, R.J. Kanzleieter, S.H. Batha, C.J. Fontes, M.E. Sherrill, D.P. Kilcrease, S.P. Regan, X-ray spectroscopic diagnostics and modeling of polar-drive implosion experiments on the National Ignition Facility, Phys. Plasmas 21 (2014) 063306.

[45] A. Mendys, M. Kański, A. Farah-Sougueh, S. Pellerin, B. Pokrzywka, K. Dzierzega, Investigation of the local thermodynamic equilibrium of laser-induced aluminum plasma by Thomson scattering technique, Spectrochimica Acta B 96 (2014) 61-68.

[46] P.R. Bevington, D.K. Robinson, Data Reduction and Error Analysis for the Physical Sciences, 3rd ed., McGraw-Hill: Boston, Massachusetts, (2003). 